\title{
Probing student reasoning approaches through the lens of dual-process theories: A case study in buoyancy
}

\author{
Cody R. Gette, ${ }^{1}$ Mila Kryjevskaia, ${ }^{1}$ MacKenzie R. Stetzer, ${ }^{2}$ and Paula R. L. Heron ${ }^{3}$ \\ ${ }^{1}$ Department of Physics, North Dakota State University, \\ 1211 Albrecht Blvd, Fargo, North Dakota 58105, USA \\ ${ }^{2}$ Department of Physics and Astronomy and Maine Center for Research in STEM Education, \\ 5709 Bennett Hall, Room 120, University of Maine, Orono, Maine 04469-5709, USA \\ ${ }^{3}$ Department of Physics, University of Washington, Box 351560, Seattle, Washington 98195-1560, USA
}

(Received 18 October 2017; published 13 March 2018)

\begin{abstract}
A growing body of scholarly work indicates that student performance on physics problems stems from many factors, including relevant conceptual understanding. However, in contexts in which significant conceptual difficulties have been documented via research, it can be difficult to pinpoint and isolate such factors because students' written and interview responses rarely reveal the full richness of their conscious and, perhaps more importantly, subconscious reasoning paths. In this investigation, informed by dualprocess theories of reasoning and decision making as well as the theoretical construct of accessibility, we conducted a series of experiments in order to gain greater insight into the factors impacting student performance on the "five-block problem," which has been used in the literature to probe student thinking about buoyancy. In particular, we examined both the impact of problem design (including salient features and cueing) and the impact of targeted instruction focused on density-based arguments for sinking and floating and on neutral buoyancy. The investigation found that instructional modifications designed to remove the strong intuitive appeal of the first-available response led to significantly improved performance, without improving student conceptual understanding of the requisite buoyancy concepts. As such, our findings represent an important first step in identifying systematic strategies for using theories from cognitive science to guide the development and refinement of research-based instructional materials.
\end{abstract}

DOI: 10.1103/PhysRevPhysEducRes.14.010113

\section{INTRODUCTION}

Over the past several decades, physics education researchers have identified student difficulties with many physics concepts and developed instructional strategies that significantly improve student performance in many contexts [1-7]. Analysis of incorrect responses often revealed patterns that may be interpreted as stemming from the students' lack of conceptual understanding. At the same time, researchers identified many contexts in which multiple refinements of instructional materials designed to address conceptual difficulties did not necessarily lead to significant improvements in student performance. A number of recent investigations have revealed that some patterns of persistent incorrect responses may be due to reasoning difficulties rather than to a lack of relevant

Published by the American Physical Society under the terms of the Creative Commons Attribution 4.0 International license. Further distribution of this work must maintain attribution to the author(s) and the published article's title, journal citation, and DOI. conceptual understanding [8-16]. In order to help students develop more productive thinking habits in the context of physics (and beyond), it is imperative to direct efforts toward (i) pinpointing more precisely the underlying mechanisms that may lead to observed patterns of student reasoning, both productive and unproductive, and (ii) utilizing the results of this research in order to develop instructional materials designed to enhance student reasoning skills.

In this study, we use the dual-process theories of reasoning and decision making as a lens for interpreting, in a mechanistic fashion, the observed patters of student reasoning. The topic of buoyancy is used as a context for this investigation. We propose and test several hypotheses that probe the impacts of specific factors and instructional circumstances on student tendencies to engage in the identified reasoning approaches. Finally, we describe instructional modifications, informed by research, that appear to produce positive shifts in productive reasoning in the specific context of buoyancy. The generalizability of our results as well as further directions for research and curriculum development are also discussed. 


\section{A. Theoretical framework: Dual-process theories of reasoning}

Research in cognitive psychology suggests that most general reasoning patterns result from distinct reasoning paths attributed to the interaction between two cognitive processes (process 1 and process 2) [17,18]. Process 1 (often referred to as the heuristic process) is described as quick, intuitive, and effortless; it is responsible for developing a "first impression" mental model of (or a way of thinking about) presented situations. Process 2 (often referred to as the analytic process) is slow, thoughtful, conscious, and effortful; it is capable of producing logic-based or rule-based reasoning. In articles on the heuristic-analytic theory of reasoning, Evans offers a diagram, shown in Fig. 1, that provides a visual aid for tracing and understanding various reasoning paths arising from the interaction (or lack thereof) between the two processes [18].

Once a reasoner is presented with a specific situation or a task, process 1 immediately and subconsciously develops a mental model of the situation based on prior knowledge and experiences, contextual cues, relevance, and other factors. This "first-available" mental model often represents a quick and subconscious attempt on the part of a reasoner to produce a coherent and plausible way of thinking about the situation at hand. In everyday language, it is often referred to as a "gut feeling," while in cognitive psychology the construct of the "firstavailable mental model" is used to provide a formal, operational definition of a reasoner's intuition. Once the first-available mental model is developed, it becomes available for scrutiny by the more rigorous, analytical process 2 . However, if a reasoner feels confident in the first-available mental model, the analytic process may be circumvented entirely $[19,20]$. If so, the first-available mental model yields a final response. This direct thinking path from a gut feeling to a final inference or judgment is overwhelmingly prevalent in everyday activities. In general, process 1 is fairly efficient and accurate at providing a quick assessment of familiar situations. Moreover, even

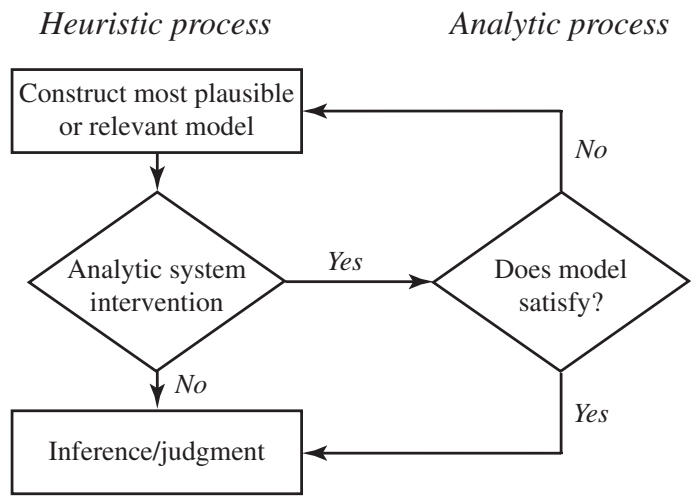

FIG. 1. Heuristic-analytic theory of reasoning and decision making [18]. if process 1 suggests an erroneous conclusion or a flawed action in an everyday domain, we are often not explicitly aware of such failures. Since we do not generally get immediate feedback on our mistakes, it is not surprising that we learn to trust our intuition, which seems to work in everyday life. As a result, novice physics learners may transfer their reliance on intuitively appealing thoughts into the context of science instruction as well. They may perceive little need for explicit and rigorous validation of their thinking.

In order to catch a mistake, the analytic process 2 must be engaged and placed on alert. If the analytic process is not satisfied with the current response, the reasoning path returns to the heuristic process, which suggests a new mental model for consideration. It is important to stress that even if the analytic process is engaged, it may be impaired by biases of its own. As such, the engagement of the analytic process does not necessarily ensure that a reasoning flaw will be detected or that a desired, logic-based argument will be generated. For example, people tend to create coherence by actively seeking confirming evidence for an existing first impression idea, a tendency referred to as confirmation bias [21]. In contrast to good scientific thinking, reasoners sometimes fail to spontaneously search for alternative mental models or counterarguments that could potentially falsify their original predictions. Therefore, even when the analytic process has been engaged, a first impression, intuitive response often still emerges as the final answer.

In summary, according to dual-process theories of reasoning, our first assessment of a situation at hand occurs through the eyes of the quick and subconscious heuristic process, which cannot be turned off. We develop first impression mental models, often without explicit awareness of thinking processes, and we often construct an argument to support the conclusion suggested by that intuitive model. The intuitive thinking process, which operates based on prior experiences and contextual cues (rather than formal reasoning), creates bias that interferes with the ability of the rational analytic thinking process to construct a valid logical reasoning chain. Many recent findings in PER are consistent with the reasoning paths accounted for by dual-process theories of reasoning. Indeed, it has been found that many students apply correct conceptual knowledge in a selective manner. They appear to be able to employ correct conceptual understanding in order to construct an argument supporting highly plausible and correct conclusions while neglecting to utilize the same correct conceptual understanding in order to refute highly plausible and erroneous conclusions [15,22,23]. We hypothesize that some persistent student difficulties identified by research may not necessarily be due to a lack of conceptual understanding, but rather may stem from basic reasoning difficulties (e.g., confirmation bias).

Physics education researchers have begun to use knowledge of implicit (i.e., heuristic) processes in order to 
identify more precisely factors that impact student reasoning in the context of physics $[8,9,14,22-24]$. One such process is related to the fluency heuristic, which is linked to processing time and provides the following mechanism for cueing a specific, first-available mental model: the faster an idea is processed, the more "weight" it is given in reasoning [25-27]. Research by Heckler and Scaife on the impact of the fluency heuristic on response patterns is of particular relevance [23]. Heckler and Scaife operationally defined and measured fluency as the time needed to arrive at an answer. They considered a class of tasks that contained competing relevant and irrelevant information. By comparing the time needed to process the given information, they determined that the relative fluency of irrelevant vs relevant information had a significant impact on answer patterns. Related to the concept of fluency is the theoretical idea of accessibility. Specifically, accessibility refers to the extent to which concepts are at the forefront of one's mind and are therefore likely to be used when making judgments [28-31]. Both concepts refer to the ease or difficulty with which information comes to the mind; however, while fluency pertains to the speed of processing (e.g., height might be processed faster than slope), accessibility refers to the likelihood that information comes to mind and is perceived to be relevant (i.e., is used in building an argument).

For the purpose of curriculum development, we are mainly concerned with improving student ability to recognize and apply relevant information. As such, in this study, we use the framework of accessibility rather than fluency. Specifically, we aim to explore the utility of dual-process theories of reasoning (DPTOR) and the theoretical idea of accessibility as a guide for interpreting student reasoning in physics and for designing interventions aimed to help students reason more productively. We propose and test several hypotheses motivated by empirical observations of both student reasoning and the idea of accessibility, which acts as a mechanism to initiate a particular reasoning path. The accessibility of an idea is influenced by many factors, including the salience of problem features and prior knowledge and experiences. In this study, we operationally define an idea to be more accessible if (i) it is cued through surface features of a task or it appears to be more familiar through prior knowledge or an explicitly stated rule and (ii) the application of the idea results in a shorter path to the answer (i.e., fewer steps) or requires rather straightforward "rulebased" reasoning (e.g., if ..., then...). It is important to note that we do not measure the accessibility of an idea directly. Instead, we use this theoretical framework to propose a hypothesis. We then infer the accessibility of an idea based on the relative prevalence of the idea in student responses. In particular, we argue that many individual students in each class have more or less the same set of available ideas and that, if we assume that each student's response is a reflection of which of those ideas is most accessible for him or her, then the prevalence of particular responses in the group is a measure of which ideas are most accessible.

\section{B. Motivation for 5-block buoyancy problem as a reasoning context}

The "5-block" buoyancy problem was designed and extensively used in an investigation of student understanding of buoyancy conducted by Loverude et al. The researchers identified a number of persistent difficulties with concepts and principles related to sinking and floating and then used the result of their investigation to develop a tutorial on buoyancy included in Tutorials in Introductory Physics by the Physics Education Group at the University of Washington (UW PEG) [4,5,32].

On the 5-block problem, students are asked to consider five blocks of the same size and shape, but different mass, as shown in Fig. 2. In the diagram, the blocks are numbered
Five blocks of the same size and shape but different masses are shown at right. The blocks are numbered in order of increasing mass (i.e., $m_{1}<m_{2}<m_{3}<m_{4}<m_{5}$ ).

All the blocks are held approximately halfway down in an aquarium filled with water and then released. Block 2 barely floats and block 5 sinks. (The final positions of blocks 2 and 5 are shown below right.)

1. In the diagram below right, sketch the final positions of blocks 1, 3, and 4. (Assume that the water is incompressible.)

2. Explain why you drew block 1 where you did.

3. Explain why you drew block 3 where you did.

4. Explain why you drew block 4 where you did.

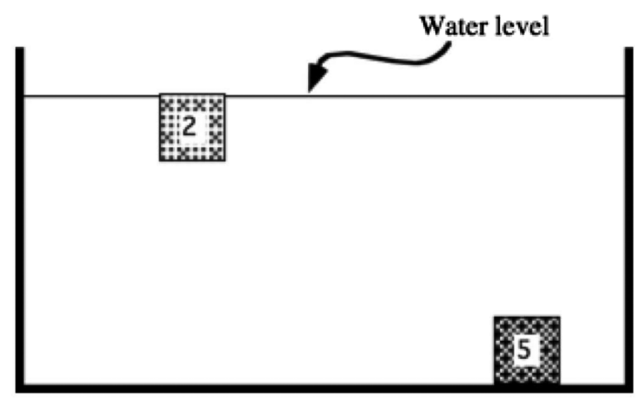

FIG. 2. The original 5-block problem. 


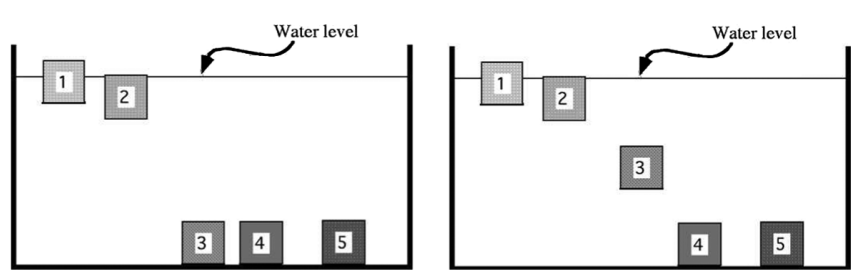

FIG. 3. Responses to the 5-block problem. Considered to be correct.

in order of increasing mass. The students are told that the blocks are held halfway down in the tank and then released. The final positions of blocks 2 and 5 are given. The students are asked to sketch the final positions of the remaining blocks and to explain their reasoning. In order to answer correctly, students may take two approaches.

- The forces argument requires students to recognize that, at the position shown, the buoyant force on block 2 is approximately equal to the weight on the block $\left(m_{2} g\right)$. Students then must compare the buoyant forces on each block just after it is released (also equal to $m_{2} g$ ) to the weight of the block. This approach is illustrated by the following student response: "[Block 3] is heavier and more dense than 2. If 2 is barely floating and 3 has a greater gravitational force than 2 and the same buoyant force, it will have a net force down." By similar reasoning, Block 1 floats while block 4 sinks.

- The density argument, on the other hand, requires students to recognize that because block 2 "barely floats" the density of the block is approximately equal to that of water. Students must compare the densities of each block to that of block 2 (and therefore to that of the water) in order to predict the sinking or floating behavior. Since the density of block 1 is less than that of block 2, block 1 will be floating as shown in Fig 3 . By similar reasoning, the densities of blocks 3 and 4 are greater than that of block 2, and blocks 3 and 4 must therefore sink. One example of a student response to the placement of block 3 , also considered to be correct, is illustrated by the quote, "Since the densities are increasing by unknown increments, one can assume 3 is either the exact same density as water, leaving it suspended or more dense, causing it to sink."

The most common incorrect student response involves the blocks floating in a "descending line": block 1 floats higher than block 2, block 3 somewhat lower than 2, and block 4 somewhat lower than block 3 (see Fig. 4). Neither 3 or 4 are on the bottom of the tank; instead, they are suspended near the middle. We hypothesized that responses of this nature may not necessarily be due to shortcomings in student conceptual understanding. We argue that some students may not even attempt to reason with formal knowledge acquired as a result of instruction. Instead, they

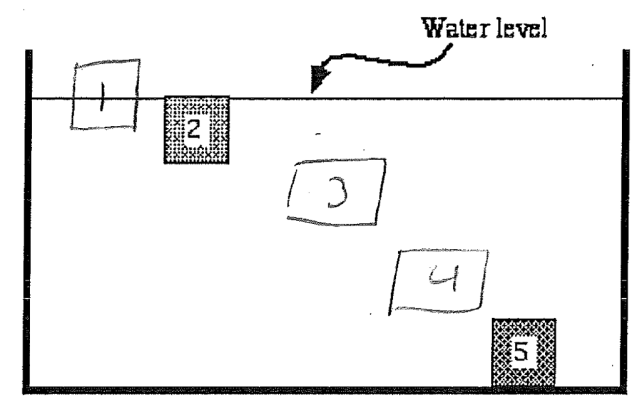

FIG. 4. Incorrect, descending line response.

may engage in intuition-based arguments cued by specific features of the questions or by their perceptions of how things work in everyday life. The possibility that many incorrect responses to the 5-block problem "were attempts to justify a prediction based on intuition" was also suggested by Loverude et al. for further investigation $[4,33]$. In the current study, we build on this prior research in order to examine the origins of relevant student intuitions and to probe the impacts of such intuitions on student reasoning.

\section{INSTRUCTIONAL CONTEXT AND STUDY DESIGN}

\section{A. Original buoyancy tutorial}

The buoyancy tutorial designed by UW PEG focuses on student understanding of forces and pressure, and on the application of these ideas to investigate the phenomenon of buoyancy [5]. The tutorial was explicitly designed to help students develop a robust understanding of the buoyant force in the context of sinking and floating. The idea that the density of an object could also be used as a predictor of sinking and floating was not explicitly discussed in the tutorial activities, but was included in the homework. A brief description of the tutorial activities is included in Appendix A.

\section{B. Instructional context}

This study was conducted in an introductory calculusbased physics course for science and engineering majors at North Dakota State University (NDSU). Over the five semesters of the study, the course was taught by two different instructors, both of whom implemented activelearning techniques in a large-enrollment lecture environment ( $N \sim 60$ students). The majority of the students were also enrolled in a weekly, fairly traditional, two-hour laboratory; however, student difficulties with the concepts of sinking and floating were not explicitly targeted during lab instruction. In all semesters, instruction on buoyancy was based on the activities from the UW tutorial. However, various modifications to the original buoyancy tutorial were implemented as part of this study, as described later in the article. 


\section{Overview of the study}

Student reasoning approaches were probed in a threepart investigation.

- In Part 1, student written responses to the 5-block problem were collected and "think aloud" interviews were also conducted. Reasoning approaches were analyzed through the lens of DPTOR and the framework of accessibility.

- In Part 2, two experiments were conducted in order to probe the impact of changes in the task design on student reasoning.

- In experiment 2.1, the 5-block problem was redesigned to reduce the salience of specific surface features thought to cue the descending line response. We hypothesized that these changes would reduce the accessibility of the unproductive descending line reasoning path.

- In experiment 2.2, the 5-block problem was redesigned to shift the salience from mass to density. We hypothesized that shifting the focus of the original 5-block problem from the ranking of masses of the blocks to the ranking of densities of the blocks would increase the accessibility of the density argument and thus increase the fraction of correct responses.

- In Part 3, two further experiments were conducted in order to probe the impact of targeted instruction on student reasoning approaches.

- In experiment 3.1, the original UW tutorial was modified to include explicit density instruction. We hypothesized that modifications that include explicit instruction on density would increase the accessibility of the density argument even further, thereby improving student performance.

- In experiment 3.2, further instructional modifications were made in order to address incorrect student intuitions about sinking and floating from everyday life. We hypothesized that such instruction would reduce the appeal of the incorrect firstavailable model, and thus make it less likely that students would bypass the analytical process. At the same time, we speculated that the increased emphasis on density would increase the likelihood that students' resulting formal reasoning would be based on density, and, given that it is easier to reason this way, improve performance.

While various conditions were tested in each experiment, a sequence of three screening questions was developed and employed as an invariant measure of student thinking throughout the study. The screening questions were designed to probe student understanding of the concepts necessary to arrive at a correct answer to the 5-block problem using the force argument. In addition, comparisons of student performance on the screening questions under different experimental conditions throughout the study helped us ensure that the instructional modifications implemented here did not impair the development of student understanding achieved by the original tutorial. A description of the screening questions is included in Appendix B.

\section{PART 1: PROBING STUDENT REASONING APPROACHES TO THE 5-BLOCK PROBLEM}

One of the goals of part 1 was to analyze student reasoning approaches (through both written exam responses and think-aloud interviews) through the lens of DPTOR. The second goal was to establish a baseline performance measure on the screening sequence and the 5-block problem at NDSU.

\section{A. Methodology}

The original UW tutorial was implemented by an instructor in the interactive lecture-based format. The instruction was entirely focused on the buoyant force; no instruction on density as a predictor of sinking and floating was formally provided in the course. The screening sequence and the 5-block problem were administered on the final exam upon completion of the original UW tutorial on buoyancy $(N=66)$. In addition, a number of student interviews were conducted prior to formal instruction on buoyancy in the introductory calculus-based physics course. There was no overlap between students participating in the interviews and answering the questions on the final exam because the interviews and final exam took place in separate semesters. The interviews followed a semistructured think-aloud protocol during which a single student was presented with the 5-block problem and was asked to predict the positions of the blocks while verbalizing his or her thoughts. In this section, we first present student interviews and then discuss student written responses to the screening sequence and the 5-block problem administered on the final exam.

\section{B. Results}

The think-aloud interview responses to the 5-block problem provided evidence that student intuition plays a role in producing the descending line response. Below, we present results from two illustrative student interviews.

Interview 1:

(1) S: [Reading question] "In the diagram below, sketch the final position of blocks 1, 3, and 4. Assume that the water is incompressible."

(2) S: "Ok, I'm basically just going off of mass and what I know from chemistry about density. Block 1 is lighter than block 2 so it's going to float a little bit more. And then block 3 is heavier than block 2, so it's going to be a little bit lower, and then lower for 4."

(3) I: "Ok, so for the first one, why did you draw block 1 where you drew it?" 
(4) S: "Because it weighs less than block 2, and block 2 is barely floating, so I drew block 1 as floating 'more' I guess."

(5) I: "Ok, and what about 3 and 4? Why did you draw them where you put them?"

(6) S: "Umm... that's a great question. I'm just guessing 'cause they weigh more than block 2 but less than block 5? So they'd be somewhere in there? Either that or they'd be at the bottom. Because they'd weigh... I don't know I'm not sure."

(7) I: "So, thinking about the physics behind it, what would cause block 4 to be lower or block 3 to be lower, or for them to be at the bottom, or at the top? How would you go about figuring that out?"

(8) S: "Well, is this like a density thing?"

(9) I: "You tell me."

(10) S: "Kind of?"

(11) I: "How would thinking about densities help?"

(12) S: "Because if it's about densities, then if it's less dense than water, it would be on top, if it's more dense than water then it would be on the bottom."

(13) I: "OK sorry, what did you say? Repeat it one more time."

(14) S: "If it's less dense than water it would be at the top. If it's more dense than water it would be at the bottom. I think. Maybe."

(15) I: "So where does that information come from, or how do you know that?"

(16) S: "Kind of from chemistry a little bit?"

(17) I: "So then basing it off what you just said, can you justify putting blocks 3 and 4 and 1 where you did?"

(18) S: "Not really. Guess I'm changing my mind."

(19) I: "Ok, changing your mind how?"

(20) S: "Now I want to keep 1 at the top and 3 and 4 at the bottom. Maybe? But I also don't know if 3 and 4 are more dense than water. But I'm thinking you can kind of assume because 3 is heavier than 2 and 2 is barely floating, but I don't know if you can actually say that."

The exchange above suggests that the student immediately recognized the relevance of both mass and density to the outcome of the presented situation (line 2). The student's first response, however, was entirely based on the ranking of the mass or, more specifically, on the notion that the ranking of the mass predicts an identical pattern in the ranking of depth. In fact, the student sketched the descending line of blocks right away, before attempting to articulate her reasoning or any kind of rule. This observed behavior is consistent with DPTOR, which suggests that, in many situations, an answer comes to mind first and only then an argument is built to support what is already believed to be true. In addition, the student seemed to view mass and density as competing variables, thus failing to recognize that, since the volume of the blocks is the same, both arguments must lead to the same prediction. Even after the relevant density rule surfaced, the student appeared to be struggling with committing to a specific reasoning approach without deciding first what the problem is "about": "[...] if it's about densities, then [...]" (lines 8-12). The student initial response appears to be consistent with the notion that the problem is "about mass".

Note that one of our explicit goals is to view student responses through the lens of DPTOR. However, the interview responses above could also be interpreted through other theoretical frameworks such as "framing" or "resources." Framing is based on a learner's perception of "what a problem is about" and is linked to the learner's prior experiences and expectations [10,11]. Similarly, the theoretical framework of "resources or knowledge in pieces" suggests that student selectiveness in choosing an argument is cued by specific contextual features $[12,13]$. We argue, however, that the framework of the DPTOR encompasses the theoretical underpinnings of both framing and resources $[9,17]$. Through the lens of the DPTOR, the subconscious and automatic selection of a "frame" or a particular "resource" occurs through the heuristic process based on prior experiences, contextual cues, expectations, and other factors.

Interview 2:

(1) S: [Reading question] "In the diagram below, sketch the final position of blocks 1,3, and 4. Assume that the water is incompressible."

(2) S: "Alright, so for block 1 I would think it would be above block 2 because it weighs less. It has less gravitational force on it. So I would put it above block 2 somewhere. Then, we know that block 5 is at the very bottom of the pool, so 3 and 4 are probably in between them [blocks 2 and 5]. 4 weighs more than 3 so I think 4 is probably going to go further down and could be at the bottom. We don't know necessarily, but I would assume it's not."

Postinterview, after answer has been discussed:

(3) I: "What did you think about [the 5-block] problem?"

(4) S: "I thought it was...I mean I didn't really know anything about the buoyancy force, so it was a little unfair. I thought it was really interesting though, because you wouldn't think that every block would sink the same even if it had different masses, so you'd think they'd be in different positions in the water, but they aren't. I guess I kind of thought of submarines and how it's harder to go down farther."

The second interview provides further evidence that a direct mapping of the ranking of the masses to the ranking of depths is readily available and appears to be subconsciously appealing. In addition, the interview debriefing revealed that this notion may be rooted in student observations that floating at different levels underwater is a common everyday occurrence, given the behavior of submarines and fish. It appears that without a robust understanding of how real entities (e.g., submarines) maintain neutral buoyancy, students may be surprised that 
TABLE I. Student performance on the screening sequence and versions of the 5-block question over the five semesters of this study.

\begin{tabular}{|c|c|c|c|c|c|}
\hline Experiment & $\begin{array}{l}\text { Modification } \\
\text { to original } \\
\text { UW tutorial }\end{array}$ & $\begin{array}{c}\text { Performance on } \\
\text { screening sequence } \\
\text { (percentage } \\
\text { correct) }\end{array}$ & $\begin{array}{c}\text { Version of } \\
\text { 5-block question }\end{array}$ & $\begin{array}{l}\text { Performance on } \\
\text { 5-block question } \\
\text { (percentage } \\
\text { correct) }\end{array}$ & $\begin{array}{l}\text { Percentage of } \\
\text { correct responses } \\
\text { supported by a } \\
\text { density argument }\end{array}$ \\
\hline Part 1: $(N=66)$ & None & 44 & Original & 62 & 38 \\
\hline \multicolumn{6}{|l|}{ Part 2: } \\
\hline $\begin{array}{l}\text { Experiment } 2.1 \\
\qquad(N=60)\end{array}$ & None & 38 & $\begin{array}{c}\text { Surface features are } \\
\text { modified }\end{array}$ & 50 & 19 \\
\hline $\begin{array}{l}\text { Experiment } 2.2 \\
\qquad(N=49)\end{array}$ & None & 39 & $\begin{array}{c}\text { Blocks are ranked by } \\
\text { density instead of } \\
\text { by mass }\end{array}$ & 51 & 88 \\
\hline \multicolumn{6}{|l|}{ Part 3: } \\
\hline $\begin{array}{l}\text { Experiment } 3.1 \\
\qquad(N=86)\end{array}$ & Density additions & 35 & Original & 57 & 65 \\
\hline $\begin{array}{l}\text { Experiment } 3.2 \\
\qquad(N=72)\end{array}$ & $\begin{array}{l}\text { Density and neutral } \\
\text { buoyancy additions }\end{array}$ & 35 & Original & 74 & 15 \\
\hline
\end{tabular}

in the case of ordinary objects (e.g., solid blocks), achieving neutral buoyancy is a very rare phenomenon.

Student written responses to the screening sequence and the 5-block question administered on exams provide further evidence that intuition plays a significant role in producing the descending line response. Postinstruction, the majority of students were able to answer each of the questions in the screening sequence with $83 \%, 66 \%$, and $65 \%$ correct, respectively, on the three questions. Only $44 \%$ of students correctly answered all three screening questions with correct and complete reasoning, thereby demonstrating that they possessed the formal knowledge and skills necessary to make a correct prediction on the 5-block problem using the force argument. Slightly less than two-thirds (62\%) of all students answered the 5-block problem correctly with complete reasoning. Of these students, $62 \%$ applied the force argument, while the rest (38\%) used the density argument, as shown in Table I. It is important to note that a considerable fraction of the students utilized the density argument in arriving at the correct prediction even though no explicit instruction on density as a predictor of sinking and floating was included in the tutorial. This pattern is consistent with the results reported by Loverude et al. Moreover, a large fraction of students ( 30\%) who correctly applied conceptual knowledge of the buoyant force to the screening questions abandoned this line of reasoning in favor of the densitybased argument on the 5-block problem. Through the lens of DPTOR, student use of the density argument without explicit instruction suggests that this rule-based argument may be more readily available (i.e., accessible) to students than the multistep force argument. As a result, the reasoning process of some students may start along the dimension of density as opposed to forces.

Further analysis of student reasoning approaches showed that about $20 \%$ of those students who used correct reasoning on all of the screening questions provided the descending line response on the 5-block problem. The following student response illustrates this inconsistency in reasoning. On the screening sequence, the student correctly sketched a free-body diagram and provided the following answers to the remaining two questions:

Screening question 2 response:

"The buoyant force on block $A$ is less than the magnitude of the buoyant force on block B. Each block has the same volume and mass, but the volume displaced by block two [sic] is greater (Archimedes' principle)."

Screening question 3 response:

"The magnitude of the buoyant force on block B is equal to that of block $C$ because they both displace the same amount of water."

On the 5-block question, however, the student did not apply the same relevant concepts. Instead, the student sketched the descending line of blocks and stated the following when justifying the locations of the blocks:

"Block 1 is the lightest. Since Block 2 barely floats, it is safe to assume that Block 1 will be floating more. Block 3 is less than Block 2 but greater than 4 and 5, so putting block 3 submerged in the water, but closer to the surface than the bottom is a good assumption. Block 4 is just a little lighter than block 5, so we can assume that it is very close to the bottom of the water, but not touching the bottom."

It appears that the student did not attempt to apply the same formal reasoning that was applied to the screening questions. Instead, the student reasoning may have been cued by the provided ranking of the blocks according to mass. Since the intuitive notion that the lightest block 1 will 
be floating on the surface is consistent with the formal reasoning, the positions of blocks 1,2 , and 5 might strongly cue the idea that the ranking of the depths of the blocks is determined by the ranking of the corresponding masses. As such, according to DPTOR, a student may not even engage the analytical process in order to check for consistency between the intuitively strong notion of the descending line of blocks and the formal knowledge of buoyancy.

A similar pattern of responses was exhibited by other students who correctly answered the screening questions, but failed to make correct predictions on the 5-block problem. These responses show that despite student ability to systematically analyze the three screening questions and to articulate correct understanding of the relevant concepts, one in every five students did not apply that same understanding on the 5-block problem; this is consistent with results obtained in other contexts [8,9]. Much like in the student response discussed above, many responses did not provide any evidence that the students had attempted to apply relevant concepts to the 5-block problem. We speculated that the descending line response is likely due to features of the questions cueing this intuitive, highly plausible (but incorrect) response. The high accessibility of such a compelling response may result in students feeling that formal support for their answers is unnecessary because the predicted outcome is "intuitively obvious."

\section{Summary}

After instruction, written exam responses fell into one of the three categories: (i) correct answers supported by the density argument, (ii) correct answers supported by the force argument, and (iii) incorrect descending line responses, with approximately $5 \%$ of responses not fitting into any of these three categories. Data indicate that even students who demonstrated conceptual understanding of the buoyant force on the screening questions did not always employ this understanding on the 5-block problem. Instead, many of the students used the (correct) density argument even without explicit instruction, while another significant fraction of the students seemed to rely on the incorrect but intuitively appealing notion that the ranking according to mass is directly mapped to the ranking according to depth. We hypothesized that such inconsistencies in student reasoning approaches are due to the heuristic process subconsciously choosing a more accessible reasoning path on the 5-block problem, typically involving either density or a mapping of the mass ranking rather than an application of the buoyant force (which seems to be less readily available). Responses involving a mapping of mass to depth are usually not justified by formal knowledge developed as a result of instruction. Instead, such responses may be rooted in incorrect student perceptions about sinking and floating in the real world, and students may therefore fail to perceive a need for any kind of rigorous justification of their thinking.

\section{PART 2: IMPACT OF CHANGES IN THE PROBLEM DESIGN ON STUDENT REASONING}

\section{A. Experiment 2.1: Modifications of the 5-block problem by removing prominent cueing features}

Prior research has suggested that the salient surface features of a task may distract from formal reasoning paths and cue intuitive responses $[8,9,15,16]$. We hypothesized that removing specific distracting features from the 5-block question may decrease the accessibility of the descending line response and result in improved student performance on the modified questions. In the new version of the 5-block problem shown in Fig. 5: (i) the ascending ranking of the masses was removed, (ii) the blocks and their relative masses were introduced one by one to give students an opportunity to consider each block individually, (iii) the two blocks with the known sinking or floating behavior were placed next to each other in order to eliminate the space for a "fill in the blanks" descending line of blocks. The task of sketching the final positions of the blocks remained the same and the sequence of screening questions remained unchanged.

\section{B. Results}

Student performance on the screening questions showed that approximately equal fractions of students were able to answer the set of screening questions correctly in both administrations, as shown in Table I. Analysis of student responses on the two versions of the 5-block problem also did not reveal any significant differences in student performance: (50\% correct in Experiment 2.1 vs $62 \%$ correct on the original problem; Fisher exact $p=0.2$ ). As in part 1, even those students who demonstrated that they possessed formal knowledge of the buoyant force on the screening questions did not apply this knowledge on the modified 5-block problem (35\% gave the descending line response after answering all screening questions correctly). These results suggest that the removal of distracting features of the problem, particularly those hypothesized to elicit and/or facilitate a descending line response, did not appear to mitigate the intuitive appeal of that response for some students.

\section{Experiment 2.2: Modifications of the 5-block problem by shifting focus from mass to density}

In experiment 2.2, students considered a question sequence identical to the original 5-block problem except the ranking of the masses was replaced by a ranking of the densities, as shown in Fig. 6. This modification was motivated by our prior results suggesting that the 
Five blocks of the same size and shape but different masses are shown at right.

\section{\begin{tabular}{l|l|l|l|l|l}
1 & 2 & 3 & 4 & 5
\end{tabular}}

All the blocks are held approximately halfway down in an aquarium filled with water and then released. Block 1 barely floats and block 2 sinks. (The final positions of blocks 1 and 2 are shown below right.)

1. Consider block 3 . The mass of block 3 is greater than the mass of block 1 and less than the mass of block 2. (i.e. $m_{1}<\boldsymbol{m}_{3}<m_{2}$ ).

Sketch the final position of block 3 on the diagram above and explain why you drew

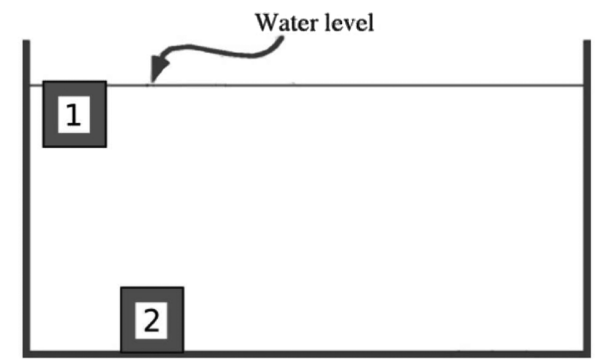
block 3 where you did.

2. Consider block 4 . The mass of block 4 less than the mass of block 1. (i.e. $\boldsymbol{m}_{4}<m_{1}<m_{2}$ )

Sketch the final position of block 4 on the diagram above and explain why you drew block 4 where you did.

3. Consider block 5 . The mass of block 5 is greater than the mass of both block 1 and block 3 , but less than the mass of block 2 (i.e. $m_{1}<m_{3}<\boldsymbol{m}_{5}<m_{2}$ ).

Sketch the final position of block 5 on the diagram above and explain why you drew block 5 where you did.

FIG. 5. The modified version of the 5-block problem in experiment 2.1: Removing salient distracting features.

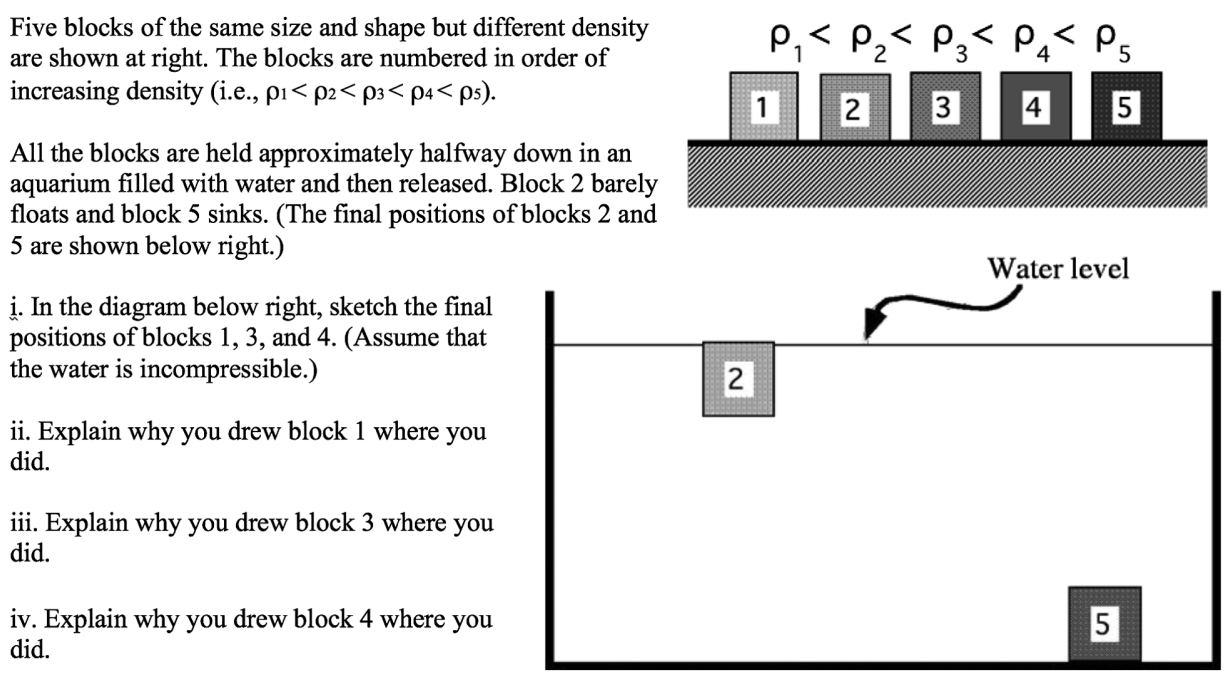

FIG. 6. The modified version of the 5-block problem in experiment 2.2: Ranking of the mass is replaced by ranking of density.

accessibility of the density argument for this student population already appeared to be high. We argued that the density argument could be categorized as "rule based": if the density of an object is higher than that of water, the block sinks; if it is lower, the block floats. This rather straightforward reasoning path (i.e., if..., then...) may be more accessible for some students than the force argument, which does not follow an algorithmic path. We hypothesized that by cueing the density of the blocks explicitly, we may increase the accessibility of the density argument even further, which may therefore increase the likelihood of students making correct predictions regarding the sinking and floating behavior of the blocks.

\section{Results}

Data analysis revealed no significant impact of the task modification on student performance: $39 \%$ of the students were correct on all screening questions, with $51 \%$ correct on the modified 5-block problem. However, an important 
but perhaps not surprising difference in the response pattern was observed: $88 \%$ of students who answered the modified 5-block problem correctly used the density argument, while the remaining $12 \%$ used the force argument. The latter responses explicitly referred to the greater mass and equal buoyant forces. These results suggest that the modified 5-block problem may have increased the accessibility of the density argument for some students. At the same time, it did not remove the intuitive appeal of the descending line of blocks argument for a large fraction of students.

\section{E. Summary}

The prevalence of the descending line responses does not appear to be impacted by altering the prominent features of the 5-block problem. In addition, even without explicit instruction, the correct density argument is common and it becomes even more prevalent when density is explicitly included in the prompt. Interpretation of these results through DPTOR suggests that by altering prominent features of the task, we were able to increase the accessibility of a specific correct reasoning approach (i.e., the prevalence of the density argument), but failed to decrease perceived relevance of the incorrect descending line approach enough to see a noticeable increase in correct responses. As such, our initial hypothesis that the heuristic process cues the persistent descending line response based on the features of the task does not find substantial support in the collected data. Specifically, it seems that while student arguments can be affected by changing the prompt, increasing the performance on the problem may require modification to instruction. As a result, we then decided to explore an additional set of hypotheses related to relevant instruction.

\section{PART 3: IMPACT OF INSTRUCTION ON STUDENT REASONING AND PERFORMANCE}

We tested the impact of two sets of instructional modifications designed based on the following justifications. In experiment 3.1, we drew on research by Heckler and colleagues that examined the impact of competing variables on student reasoning. They discovered that (i) providing students with a correct rule for predicting an outcome reduces reliance on an irrelevant competing variable, and (ii) providing opportunities for practice decreases the response time, thereby promoting the likelihood that a specific desired argument comes to mind faster. These experiments were performed in a laboratory setting and the interventions were given just before students were asked to consider a task. In our case, we probed the impact of instructional modifications on the accessibility of the density argument. We speculated that the rule-based density argument may have more perceived usability than the forces argument due to its (relatively) straightforward application. This in turn may make the density argument more competitive with the intuitive descending line idea. As such, we included supplemental exercises that (i) introduced the concept of density as a predictor of sinking and floating and (ii) strengthened the links between the force and density arguments. We hypothesized that explicit instruction on density will further increase the accessibility of the density argument and will therefore improve student performance on the original 5-block problem.

In experiment 3.2, we tested an alternative hypothesis. The results from parts 1 and 2 suggest that changes in the task design were impacting the accessibility of the correct density argument while failing to reduce relevance of the incorrect descending line approach. This suggests that the students' descending line responses may be based on strong, everyday perceptions of sinking and floating behavior, as illustrated by student interview 2 in part 1 . These students may incorrectly think that regular objects free floating under water are a common occurrence. This perception may be rooted in student observations of fish, submarines, and other objects and animals that can adjust their average densities so as to become neutrally buoyant. For these students, the outcome of regular objects (such as solid blocks) floating at different depths below the water surface may be perceived to be common and therefore highly plausible. While formal instruction on buoyant forces may have helped these students develop the conceptual understanding necessary to answer the 5-block problem correctly, these students may still have perceived little need to apply such understanding to justify predictions based on their intuitive ideas. If, indeed, the descending line responses are strongly cued by student intuitions about how "real world" works, then the instructional modifications proposed in experiment 3.1 are also not likely to produce significant shifts in student performance. We hypothesized that for the students who answer the 5-block problem correctly, the plausibility of objects freefloating under water may not be as strong. Therefore, these students may be more likely to apply formal reasoning to arrive at a correct answer. For this category of student thinking, the inclusion of explicit instruction on density is likely to further increase the accessibility of the density argument and, as such, increase the prevalence of this argument on the 5-block problem. (This is consistent with the shifts in the prevalence of the density arguments in experiments 2.1 and 2.2.)

Finally, we hypothesized that if the nature of the descending line responses is linked to student perceptions about the behavior of fish and submarines, then, in order to decrease the accessibility of such responses, further instructional modifications designed to highlight the complex sequence of steps required to achieve neutral buoyancy at different depths are required. In order to test this hypothesis, in experiment 3.2, we modified 
instruction by implementing a set of questions designed to guide students through the reasoning steps necessary to understand the conditions that allow a submarine to float under water and to change its depth. We also included hands-on, in-class activities that allowed students to experience firsthand the sinking and floating behavior of common objects. We hoped that the students would realize that it is extremely unlikely to observe common objects floating under water and it is also extremely difficult to achieve neutral buoyancy in common laboratory settings. We hoped that these experiences would help students recognize that the descending line outcome of the 5-block problem is highly unlikely, which in turn may prompt students to consider alternative outcomes by applying formal reasoning approaches.

\section{A. Experiment 3.1: Explicit instruction on density as a predictor of sinking and floating}

Supplemental exercises focusing on density as a predictor of sinking and floating. Two exercises were designed to supplement the development of ideas in the original UW tutorial. The first exercise was introduced immediately after the discussion of the buoyant force. Students were asked to consider three objects of the same shape: two blocks of different density and a cubical volume of water with an imaginary boundary around it. Students related the sinking and floating behavior of the blocks to the density of the blocks relative to that of the water. The students were then asked to articulate a simple "rule" for predicting whether an object will sink or float based on its density. A second supplemental exercise was included at the end of the tutorial. The students were asked to predict the sinking or floating behavior of a block and support their predictions by applying both the force and the density arguments.

\section{B. Results}

The screening sequence and the original 5-block problem were used to examine the impact of the instructional modifications designed to increase student accessibility with relevant concepts of density and buoyant force. Analysis of student responses did not reveal any significant improvement in student performance. However, as in experiment 2.2, the prevalence of the density-based correct responses increased substantially compared to that in part 1 . Respectively, $62 \%$ and $57 \%$ of students answered the original 5-block problem correctly in part 1 and in experiment 3.1. Of these correct responses, $38 \%$ used the density argument in part 1 compared to $65 \%$ in experiment 3.1 (see Table I). This suggests that, as a result of instruction, the accessibility of the density argument was increased, for at least some fraction of students. At the same time, for those students who answered the 5-block problem incorrectly, the possible increase in the accessibility of the density argument was not enough to override the intuitive appeal of the descending line prediction. This result is consistent with the alternative hypothesis above used to justify experiment 3.2.

\section{Experiment 3.2: Including hands-on and real world examples in instruction}

In this study so far, we have shown that changing the design of the 5-block problem did not significantly impact student performance, thereby suggesting student intuition is not cued by salient features in this particular case. We have also shown that instructional changes targeting the density argument did not significantly improve performance. Evidence from student interviews suggests that students tend to draw upon intuition involving real world situations (submarines, fish, etc.) in which "floating in the middle" is common. This is further supported by Heron et al. who reported that significant improvement on the 5-block problem was achieved only after implementation of lengthy experimental lab activities that included instruction on density [5,33]. In experiment 3.2 , we modified the instructional sequence used in experiment 3.1 in order to (i) include explicit hands-on activities to help student recognize that neutral buoyancy is difficult to achieve, (ii) give students opportunities to analyze conditions necessary for objects of variable average density (e.g., submarines) to achieve neutral buoyancy, and (iii) help students articulate the steps necessary for a submarine to move between different depths. We hypothesized that these activities, combined with the density instruction implemented in Experiment 3.1, would decrease the accessibility of the descending line argument, which, in turn, would yield improved student performance on the 5-block problem.

Supplemental exercises focusing on hands-on activities and real-world applications. The hands-on activities in the modified tutorial followed a sequence of intellectual steps nearly identical to that contained in the sections of the original tutorial introducing the buoyant force and focusing on sinking and floating of hypothetical solid blocks. However, the modified tutorial included simple experiments instead of considering hypothetical solid blocks. Students were provided with two small test tubes, identical in shape, and a large beaker of water. The tubes were partially prefilled with different masses and a small amount of water. Students observed the motion of the tubes released under water and analyzed the forces acting on the tubes. Tube 1 floated easily above the surface, while tube 2 was very nearly neutrally buoyant and rose to the surface slowly. Students observed the behaviors of the tubes, noted the differences in their motions, and analyzed forces acting on the tubes right after they were released under water. Then, the students were asked to predict and analyze the motion of tube 2 after more mass was added to the tube. Students were given opportunities to check their 
predictions. This sequence of hands-on activities bore no resemblance to the 5-block problem.

After students analyzed the motions of the tubes using both the force and the density arguments (similar to the tutorial version in experiment 3.1), they focused on motion of objects of variable average density, such as submarines. Students were guided to articulate conditions necessary for a submarine to remain under water at rest and to move between different depths. Students were asked to use both the force and the density arguments. Finally, a hypothetical dialogue between two fictitious students was presented, which concerned two submarines that were identical in shape but floating at two different depths. Student 1 incorrectly states that the mass of the submarine at a shallower depth is less than that of the submarine deeper under water. Student 2 agrees and supports this incorrect prediction by stating that the ballast will be lower for the submarine closer to the surface. Students were expected to recognize the incorrect elements of reasoning presented in the dialogue and to justify their answers. Specifically, students must recognize that, since the buoyant force does not depend on depth, the ballast at either depth must be identical to ensure that the net force on each submarine is zero. As such, both students in the dialogue incorrectly related the mass of the submarine to the depth.

\section{Results}

The screening sequence and the original 5-block problem were again used in order to examine the impact of the instructional modifications. Performance on the screening questions was essentially unchanged, but the fraction of correct responses on the 5-block problem increased significantly when compared to experiments $2.1,2.2$, and 3.1 (74\% correct with correct reasoning; Fisher exact $p<0.05)$. While the performance was not significantly different compared to that in Part 1, the increase in performance in experiment 3.2 is statistically significant compared to all other semesters. This suggests that perhaps student performance in part 1 is an outlier. Moreover, for the purposes of this study, the increase in student performance from experiment 3.1 to experiment 3.2 is of particular importance as the instructional materials for experiment 3.2 were modified from those used in experiment 3.1 as described above. This seems to suggest that the additional instruction on real-world applications with a hands-on component had a significant impact on student reasoning compared to the previous semester. Indeed, some of the student responses to the 5-block problem directly referenced the observations made in class. For example, one student wrote

"It is possible for an object to remain in the center of the aquarium if the forces are just right, however due to the fact that [block] 2 was barely floating and completely submerged and from what I saw with the experiments in class, I felt that block 3 would sink to the bottom because the buoyant force for blocks 3 and 2 would be equal (same size and both fully submerged). The mass of block 3 is reasonably heavier so the force down by the earth would be great enough for it to sink."

\section{E. Discussion}

Several explanations are possible for the increase in performance on the 5-block problem. We argue that perhaps two factors are critical. First, the hands-on activities provided students with opportunities to observe that it is uncommon for ordinary objects to be in the state of neutral buoyancy. Second, the analysis of conditions necessary for submarines to achieve the state of neutral buoyancy and to move between different depths may have helped address incorrect student perceptions regarding sinking and floating of objects of variable densities. As such, these instructional modifications may have removed the strong intuitive appeal of the descending line response to the 5-block problem. This explanation is consistent with student responses similar to the one quoted above. Moreover, according to DPTOR, once the intuitively appealing argument is removed, the remaining possibility becomes most plausible. Since the most plausible answer is now typically consistent with the correct application of the formal knowledge acquired as a result of instruction, students are more likely to provide correct answers with correct reasoning, hence the increase in performance. Alternatively, it could be argued that perhaps the modified instruction is more effective at improving student conceptual understanding of buoyancy or provides more time on task. However, since the increase in the fraction of correct responses to the 5-block problem occurred without any improvement in student performance on the screening questions (see Table I) and the additional instructional time needed to cover neutral buoyancy was relatively minor, we are inclined to think that the modified instruction achieved exactly what it was intended to do: remove the intuitive appeal of the incorrect descending line response.

The outlined reasoning path suggested by DPTOR is consistent with an "answer first, reasoning second" pattern (or confirmation bias). As such, it could be debated whether or not the increase in student performance in experiment 3.2 is an indication of success. One could argue that although the percentage of correct responses increased, the new reasoning path is no more sophisticated than the one that led to the prediction of the descending line: both start with the most intuitively appealing outcome and lead to an answer. The key, however, is that we were able to isolate, through hypothesis testing, a specific aspect of student thinking that appears to be the source of a very persistent incorrect response shown to be particularly resistant to many research-based instructional efforts to improve student conceptual understanding. 
Moreover, according to DPTOR, reasoners see the world around them through the lens of the quick and automatic process 1. It immediately suggests the most plausible mental model of an unfamiliar situation and only then the slow and deliberate process 2 may intervene in order to provide an evaluation. In other words, intuitive thoughts cannot be turned off. Simon defines intuition as "pattern recognition" [34]. While experts possess a large repertoire of experiences that allow them to quickly and effectively recognize both correct and incorrect patterns in their thinking, novice learners lack such experiences. As a result, they are less likely to recognize instances of intuitive thoughts or biased reasoning, even when process 2 is engaged. We argue that classroom activities that give students opportunities to slow down, examine "firstavailable responses," and recognize instances of biased reasoning must be viewed as an essential component of instruction that ultimately leads to the development of expertise in physics. This research project advocates for the development of instructional materials that help students refine their reasoning approaches alongside the development of robust conceptual understanding.

\section{CONCLUSIONS}

This research was motivated by observed patterns of persistent student difficulties that have been shown to be resistant to changes in instruction. We applied dual-process theories of reasoning and decision making and the theoretical framework of accessibility in order to identify factors and instructional circumstances that enhance or suppress productive and unproductive reasoning approaches. The context of buoyancy was chosen as a case study. We hypothesized that the observed patterns of student incorrect responses were more likely to be due to incorrect but intuitively appealing student ideas rather than to a lack of conceptual understanding. Indeed, some of the students who possess the requisite knowledge of relevant ideas may not even attempt to apply this knowledge in order to arrive at an answer. Instead, these students tend to abandon formal approaches to solving a problem if that problem cues a strong intuitive response. Several specific hypotheses were tested in order to pinpoint more precisely the root of student intuitive ideas.

The first set of hypotheses probed whether or not student responses to the 5-block problem are strongly cued by prominent surface features of the task; if so, then deliberate changes in the task design were predicted to alter patterns in student reasoning.

- The experiment 2.1 hypothesis predicted that prominent surface features of the original 5-block problem strongly cued an incorrect descending line response. To test the hypothesis, several changes were made to the design of the problem; however, no significant differences in student performance were observed.

- The experiment 2.2 hypothesis predicted that shifting the focus of the original 5-block problem from the ranking of masses of the blocks to the ranking of densities of the blocks will increase the accessibility of the rule-based density argument and thus increase the fraction of correct responses. After the proposed modifications were implemented, no significant difference in the fraction of correct responses was observed. However, the accessibility of the density argument seemed to be increased among those students who provided correct answers to the problem. The results of testing the first two hypotheses suggested that the observed pattern of incorrect student responses could not be attributed to features of the task design. As such, an additional set of hypotheses was proposed that focused on probing the impact of instructional interventions, rather than the task design, on patterns of student reasoning.

- The experiment 3.1 hypothesis focused on probing whether or not instructional modifications that include explicit instruction on density will increase the accessibility of the rule-based density argument even further, thereby improving student performance.

- The experiment 3.2 hypothesis represented an alternative to the experiment 3.1 hypothesis. It was argued that perhaps the lack of impact of the increased accessibility of the density argument on the fraction of incorrect responses was due to the students' strong intuitive ideas about the presented situation itself. These intuitive ideas about how real world works (e.g., fish swimming under water and submarines moving between different depths) may be producing strong impacts on student reasoning approaches. Specifically, students may be so confident in the descending line response based on their intuitions that they may perceive little or no need to apply formal knowledge acquired as a result of instruction. As such, it was argued that instructional modifications in experiment 3.1 would produce little or no impact on student performance. The experiment 3.2 hypothesis predicted that instruction designed to specifically address student perceptions about sinking and floating of objects of variable densities such as submarines would be more likely to produce a positive shift in student performance. It is important to stress that the instructional modifications implemented as part of testing the hypotheses associated with experiments 3.1 and 3.2 were designed to supplement tutorial activities intended to help students develop conceptual understanding of buoyancy. Such understanding is critical for helping students recognize shortcomings in their intuitive ideas and for helping students resolve inconsistencies between their predictions and the observed outcomes. While instructional modifications in experiment 3.1 did not produce a measurable shift in student performance, those in experiment $3.2 \mathrm{did}$ in fact produce a significant increase in the fraction of correct responses. 
We argue that this study illustrates the importance of research aimed at identifying factors and instructional circumstances that enhance or suppress productive and unproductive student reasoning approaches. Dual-process theories of reasoning suggest that the quick and automatic process 1 cannot be turned off. As such, instruction that explicitly helps students examine their intuitive ideas is necessary to further improve student performance in physics $[35,36]$. However, we argue that the instructional modifications designed and implemented in this study produced an impact solely because they supplemented tutorial instruction already shown to be highly effective at helping students develop a formal understanding of the concepts of buoyancy.

In future work, we plan to examine this argument in more detail. In particular, we suspect that the inclusion of supplemental materials that promote student reflection on intuitive ideas are likely to be more effective if offered as a follow-up to formal instruction aimed at improving student conceptual understanding of relevant topics. In the absence of solid understanding of relevant concepts, a "mindware gap" is produced, which is challenging for the students to bridge in order to productively reconcile inconsistencies in their reasoning [37]. Systematic hypothesis testing could help us ascertain how best to help students navigate their process 1 responses.

\section{ACKNOWLEDGMENTS}

The authors would like to acknowledge Andrew Boudreaux for his contributions to and valuable discussions about this project. We also appreciate the feedback and suggestions for improving this manuscript from our anonymous reviewers. This material is based upon work supported by the National Science Foundation under Grants No. DUE-1431857, No. DUE-1431541, No. DUE1431940, No. DUE-1432765, No. DUE-1432052, and No. DRL-0962805.

\section{APPENDIX A: ORIGINAL BUOYANCY TUTORIAL}

In Sec. I, students consider the behavior of a block submerged in water. Students are told that after the block is released, it is observed to float on the surface of the water. Students are asked (i) to draw an extended free-body diagram of the block just after it is released in the middle of the beaker of water and to identify all forces, (ii) to use the relationship between pressure and depth to compare the magnitudes of the vertical forces, (iii) to determine the vector sum of the forces exerted on the block by the surrounding water and to compare this force to the weight of the block. In the following section, students are told that the experiment is repeated with a second block of the same volume and shape; however, the second block is observed to sink. Students are then guided through the same set of questions. Upon completion of this section, the buoyant force is introduced as the vector sum of the forces exerted on an object by the surrounding liquid. Students are asked to articulate explicitly whether or not the buoyant force on an object that is completely submerged in an incompressible liquid depends on the mass or weight of the object, the depth below the surface at which the object is located, and the volume of the object. Students are expected to recognize that neither the mass nor the depth of the object impacts the magnitude of the buoyant force.

In Sec. II, Archimedes' principle is introduced along with the idea of the volume of liquid displaced by the object.

Section III explicitly focuses on sinking and floating. First, students consider block $A$, which is observed to float on the surface of the water after it had been released from the center of a beaker. Students compare the buoyant force and the weight at two instances: right after block $A$ is released and when the block reaches the surface. Then, students consider a different block, block $B$, of the same size and shape as block $A$, but with a slightly greater mass. The block is observed to barely float on the surface of the water. Students compare the buoyant forces on blocks $A$ and $B$ right after they are released and at their final positions. Finally, students consider block $C$ of the same size and shape as blocks $A$ and $B$, but with a mass slightly greater than that of block $B$. Students are asked to consider a hypothetical incorrect prediction of the final position of the block given by two fictitious students and to identify incorrect elements of reasoning given by both students. Specifically, Student 1 incorrectly uses the mass of the block to predict that block $C$ will float right below the surface since block $C$ is heavier than block $B$. Student 2 agrees and supports this incorrect prediction with a force argument: since the buoyant force on block $C$ is slightly less than that on block $B$, block $C$ should come to rest a bit below the surface. Students are expected to recognize that student 2 ranked the buoyant forces on blocks $B$ and $C$ correctly, but failed to recognize that the net force on block $C$ points toward the bottom of the beaker.

\section{APPENDIX B: SCREENING QUESTIONS}

The force argument that leads to the correct answer to the 5-block problem requires the following logical steps: (i) recognize that the buoyant force is determined by the displaced volume of the liquid (and the density of that liquid) rather than by the objects' mass or depth, and (ii) draw a free-body diagram and use Newton's laws to predict the direction of the net force on the object of interest. More specifically, students must recognize that the buoyant force on all the submerged blocks is the same, while the weight increases according to the mass; since block 2 barely floats, the buoyant force on the submerged blocks is approximately equal to the weight of block 2 . 


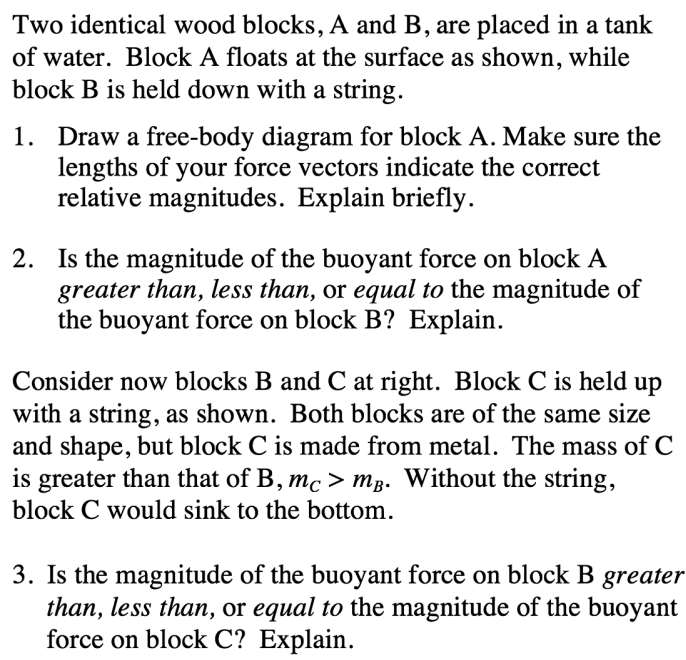
of water. Block A floats at the surface as shown, while block B is held down with a string.

1. Draw a free-body diagram for block A. Make sure the lengths of your force vectors indicate the correct relative magnitudes. Explain briefly.

2. Is the magnitude of the buoyant force on block A greater than, less than, or equal to the magnitude of the buoyant force on block B? Explain.

Consider now blocks $\mathrm{B}$ and $\mathrm{C}$ at right. Block $\mathrm{C}$ is held up with a string, as shown. Both blocks are of the same size and shape, but block $\mathrm{C}$ is made from metal. The mass of $\mathrm{C}$ is greater than that of $\mathrm{B}, m_{C}>m_{B}$. Without the string, block $\mathrm{C}$ would sink to the bottom.

3. Is the magnitude of the buoyant force on block B greater than, less than, or equal to the magnitude of the buoyant force on block C? Explain.
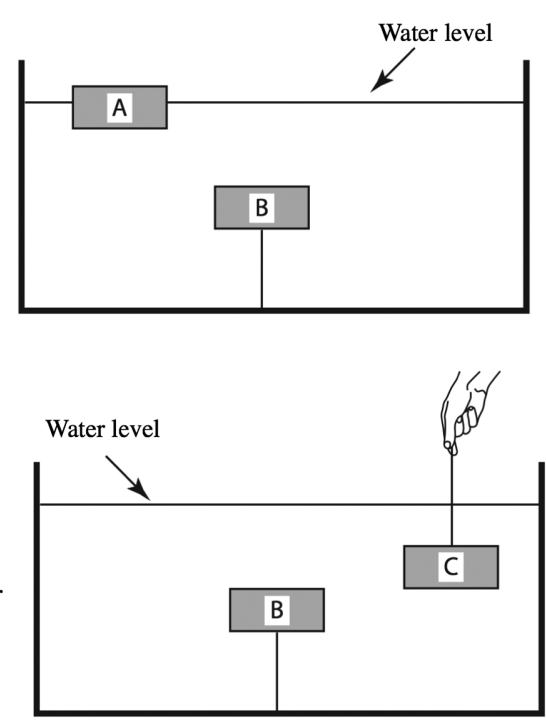

FIG. 7. Sequence of screening questions.

This suggests that the net force on block 1 is toward the surface of the liquid and block 1 will therefore float; however, the net forces on blocks 3-5 are toward the bottom of the container, so those blocks will sink. The screening questions, shown in Fig. 7, require students to demonstrate the same knowledge and conceptual understanding outlined above. In the first two questions, students are asked to consider blocks $A$ and $B$. They are told that both blocks have identical volume and mass. The first question asks students to draw a free-body diagram for block $A$, thereby identifying both forces acting on the block (mg and $\left.F_{\text {buoyant }}\right)$. The following question asks students to compare the magnitude of the buoyant force on block $A$ to that on block $B$. This question probes whether or not students recognize that, although the blocks are identical, the volume displaced by block $A$ is less than that displaced by block $B$. Therefore, the magnitude of the buoyant force on $A$ is less than that on $B$. The final question asks students to compare the buoyant forces on blocks $B$ and $C$ of equal volume, but different mass $\left(m_{C}>m_{B}\right)$. The blocks are completely submerged and suspended at different depths by two strings, as shown in Fig. 7. Students are told that in the absence of the strings, block $B$ would float, while block $C$ would sink. Once again, students are expected to recognize that only the displaced volume determines the ranking of the buoyant forces in this situation, and therefore the buoyant forces on blocks $B$ and $C$ are equal in magnitude.
[1] L.C. McDermott and E.F. Redish, Resource Letter: PER-1: Physics Education Research, Am. J. Phys. 67, 755 (1999).

[2] L. Hsu, E. Brewe, T. M. Foster, and K. A. Harper, Resource Letter RPS-1: Research in problem solving, Am. J. Phys. 72, 1147 (2004).

[3] D. E. Meltzer and R. K. Thornton, Resource Letter ALIP-1: Active-Learning Instruction in Physics, Am. J. Phys. 80, 478 (2012).

[4] M. E. Loverude, C. H. Kautz, and P. R. L. Heron, Helping students develop an understanding of Archimedes' principle. I. Research on student understanding, Am. J. Phys. 71, 1178 (2003).

[5] P. R. L. Heron, M. E. Loverude, P. S. Shaffer, and L. C. McDermott, Helping students develop an understanding of Archimedes' principle. II. Development of research-based instructional materials, Am. J. Phys. 71, 1188 (2003).
[6] M. Kryjevskaia, M. R. Stetzer, and P. R. L. Heron, Student understanding of wave behavior at a boundary: The relationships among wavelength, propagation speed, and frequency, Am. J. Phys. 80, 339 (2012).

[7] L. C. McDermott, Research on conceptual understanding in mechanics, Phys. Today 37, 24 (1984).

[8] M. Kryjevskaia, M. R. Stetzer, and N. Grosz, Answer first: Applying the heuristic-analytic theory of reasoning to examine student intuitive thinking in the context of physics, Phys. Rev. ST Phys. Educ. Res. 10, 020109 (2014).

[9] A. F. Heckler, in Psychology of Learning and Motivation (Elsevier, New York, 2011), Vol. 55, pp. 227-267, ISBN 978-0-12-387691-1.

[10] D. Hammer, A. Elby, R. E. Scherr, and E. F. Redish, Resources, framing, and transfer, in Transfer of learning from a modern multidisciplinary perspective, edited by 
J. P. Mestre (Information Age Publishing, Greenwich, Connecticut, 2005), Vol. 89, pp. 89-120.

[11] I. P. Levin, S. L. Schneider, and G. J. Gaeth, All frames are not created equal: A typology and critical analysis of framing effects, Organ. Behav. Hum. Decis. Process. 76, 149 (1998).

[12] A. Disessa, in Constructivism in the Computer Age, edited by G. Forman and P. Pufall (Lawrence Erlbaum, Hillsdale, NJ, 1988), pp. 49-70.

[13] L. Louca, A. Elby, D. Hammer, and T. Kagey, Epistemological resources: Applying a new epistemological framework to science instruction, Educ. Psychol. 39, 57 (2004).

[14] P. R. Heron, Testing alternative explanations for common responses to conceptual questions: An example in the context of center of mass, Phys. Rev. Phys. Educ. Res. 13, 010131 (2017).

[15] L. Lising and A. Elby, The impact of epistemology on learning: A case study from introductory physics, Am. J. Phys. 73, 372 (2005).

[16] M. S. Sabella and G. L. Cochran, Evidence of intuitive and formal knowledge in student responses: Examples from the context of dynamics, AIP Conf. Proc. 720, 89 (2004).

[17] D. Kahneman, Thinking, Fast and Slow (Macmillan, New York, 2011), ISBN 978-0-374-27563-1.

[18] J. S. B. T. Evans, The heuristic-analytic theory of reasoning: Extension and evaluation, Psychon. Bull. Rev. 13, 378 (2006).

[19] V. A. Thompson, J. A. Prowse Turner, and G. Pennycook, Intuition, reason, and metacognition, Cogn. Psychol. 63, 107 (2011).

[20] V. A. Thompson, J. S. B. T. Evans, and J. I. D. Campbell, Matching bias on the selection task: It's fast and feels good, Think. Reas. 19, 431 (2013).

[21] R. S. Nickerson, Confirmation bias: A ubiquitous phenomenon in many guises, Rev. Gen. Psych. 2, 175 (1998).

[22] M. Kryjevskaia, M. R. Stetzer, and P. R. L. Heron, Student difficulties measuring distances in terms of wavelength: Lack of basic skills or failure to transfer?, Phys. Rev. ST Phys. Educ. Res. 9, 010106 (2013).

[23] A. F. Heckler and T. M. Scaife, Patterns of response times and response choices to science questions: The influence of relative processing time, Cogn. Sci. 39, 496 (2015).

[24] A. K. Wood, R. K. Galloway, and J. Hardy, Can dual processing theory explain physics students' performance on the Force Concept Inventory?, Phys. Rev. Phys. Educ. Res. 12, 023101 (2016).

[25] R. Hertwig, S. M. Herzog, L. J. Schooler, and T. Reimer, Fluency heuristic: A model of how the mind exploits a byproduct of information retrieval, J. Exper. Psych. Learning Memory Cognition 34, 1191 (2008).

[26] L. J. Schooler and R. Hertwig, How forgetting aids heuristic inference, Psychol. Rev. 112, 610 (2005).

[27] B. D. Mikula and A. F. Heckler, Framework and implementation for improving physics essential skills via computer-based practice: Vector math, Phys. Rev. Phys. Educ. Res. 13, 010122 (2017).

[28] N. Schwarz, H. Bless, M. Wänke, and P. Winkielman, Accessibility revisited, in Foundations of Social Cognition: A Festschrift in Honor of Robert S. Wyer, Jr. (Lawrence Erlbaum Associates Inc, Hillsdale, NJ, 2003), pp. 51-77.

[29] E. T. Higgins, Knowledge activation: Accessibility, applicability, and salience, in Social psychology: Handbook of basic principles (US: Guilford Press, New York, NY, 1996), pp. 133-168.

[30] D. Kahneman, A perspective on judgment and choice: Mapping bounded rationality, Am. Psychol. 58, 697 (2003).

[31] C. K. Morewedge and D. Kahneman, Associative processes in intuitive judgment, Trends Cognit. Sci. 14, 435 (2010).

[32] L. C. McDermott and P. S. Shaffer, Tutorials in Introductory Physics (Prentice Hall, Englewood Cliffs, NJ, 1998).

[33] M. E. Loverude, A research-based interactive lecture demonstration on sinking and floating, Am. J. Phys. 77, 897 (2009).

[34] H. A. Simon, Invariants of human behavior, Annu. Rev. Psychol. 41, 1 (1990).

[35] R. E. Scherr and A. Elby, Enabling informed adaptation of reformed instructional materials, AIP Conf. Proc. 883, 46 (2007).

[36] T. I. Smith and M. C. Wittmann, Comparing three methods for teaching Newton's third law, Phys. Rev. ST Phys. Educ. Res. 3, 020105 (2007).

[37] K. E. Stanovich, What Intelligence Tests Miss: The Psychology of Rational Thought (Yale University Press, New Haven, CT, 2009), ISBN 978-0-300-14253-2. 